\title{
Spinal cord dysfunction caused by non-traumatic hematomyelia
}

\author{
A Karavelis, G Foroglou, A Petsanas and T Zarampoukas \\ Department of Neurosurgery, Aristotelian University of Thessaloniki, AHEPA General Hospital, 54006 Thessaloniki, \\ Greece
}

Two patients with a non-traumatic hematomyelia at $\mathrm{T}_{9}$ and $\mathrm{C}_{3}-\mathrm{C}_{7}$ respectively, are presented. Both patients presented with pain and a Brown-Sequard syndrome. The preoperative diagnosis was made by magnetic resonance imaging, while myelography and computerized tomography were not helpful. Surgical evacuation of the hematomas and in one patient removal of a vascular malformation were performed. The neurological symptoms and signs, after a temporary worsening, progressively improved. The prompt surgical treatment of this entity and the usefulness of magnetic resonance imaging in the preoperative diagnosis and on the planning of the surgical strategy is particularly emphasized.

Keywords: hematomyelia; spinal cord; magnetic resonance imaging; cryptic vascular malformation

\section{Introduction}

Non-traumatic hematomyelia is an extremely rare condition compared to intracerebral non-traumatic hematomas. They are usually due to small vascular malformations a few millimeters in diameter which were first characterized as being 'cryptic' because of the difficulty to detect them by angiography. ${ }^{1}$ Recently, using magnetic resonance imaging (MRI) it is possible to detect these cryptic vascular lesions and the associated hematoma with great accuracy. ${ }^{2-5} \mathrm{We}$ present two patients who had a 'spontaneous' hematomyelia and discuss the diagnostic, therapeutic and prognostic implications of this condition, also reviewing the relevant literature.

\section{Case reports}

Case 1

A 77-year-old male developed suddenly severe low back pain radiating to the left leg for the first time, in November 1990. One month later he experienced weakness and numbness of that leg, and after 3 months similar symptoms appeared in the right leg. $\mathrm{He}$ could not walk without assistance. On admission, neurological examination showed spastic paraparesis more evident in the left side. The tendon reflexes in both lower limbs were increased, the plantar responses were extensor. Anesthesia for light touch and temperature was present bilaterally below the $T_{12}$ dermatome. In addition there was urinary retention and constipation.

Radiological examination Plain films of the thoracic

Correspondence: A Karavelis and lumbar spine were normal. Also myelography and CT-myelogram were unhelpful. MRI revealed dilatation of the abdominal aorta and, at the level of $T_{9}$, an intramedullary mass with central low signal intensity surrounded by a zone of high and low signal on both the $T_{1}$ and the $T_{2}$-weighted images, characteristic of an intramedullary hematoma (Figure 1).

Operation The patient underwent laminectomy from $\mathrm{T}_{8}$ to $\mathrm{T}_{10}$. There was no dilatation of those segments of the spinal cord, which had the typical appearance of an underlying hematoma. Using the operating microscope a myelotomy was performed which revealed a single cavity containing old blood. After irrigation we did not observe any abnormal vessels and there was no active bleeding. The patient made a good recovery although in the 3 days after operation there was transient neurological worsening, motor function of the lower extremities improved over the following months. On follow up, 6 months later, he could walk with the aid of a walker. There was mild spasticity, mainly of the left leg, which was successfully managed with baclofen.

Case 2

A 21-year-old woman had acute pain in the neck and the right scapula 15 days before admission. At the time of admission neurological examination revealed a markedly spastic gait, and loss of position, touch and vibratory sense in the upper extremities and to a minor degree in the lower extremities. Pain and temperature sensation was decreased below the $\mathrm{C}_{6}$ dermatome mainly on the left side. There was moderate weakness in all four extremities more prominent in the right side, with a Brown-Sequard type syndrome. In addition, there was bladder and bowel dysfunction.

Radiological examination A Metrizamide myelogram 


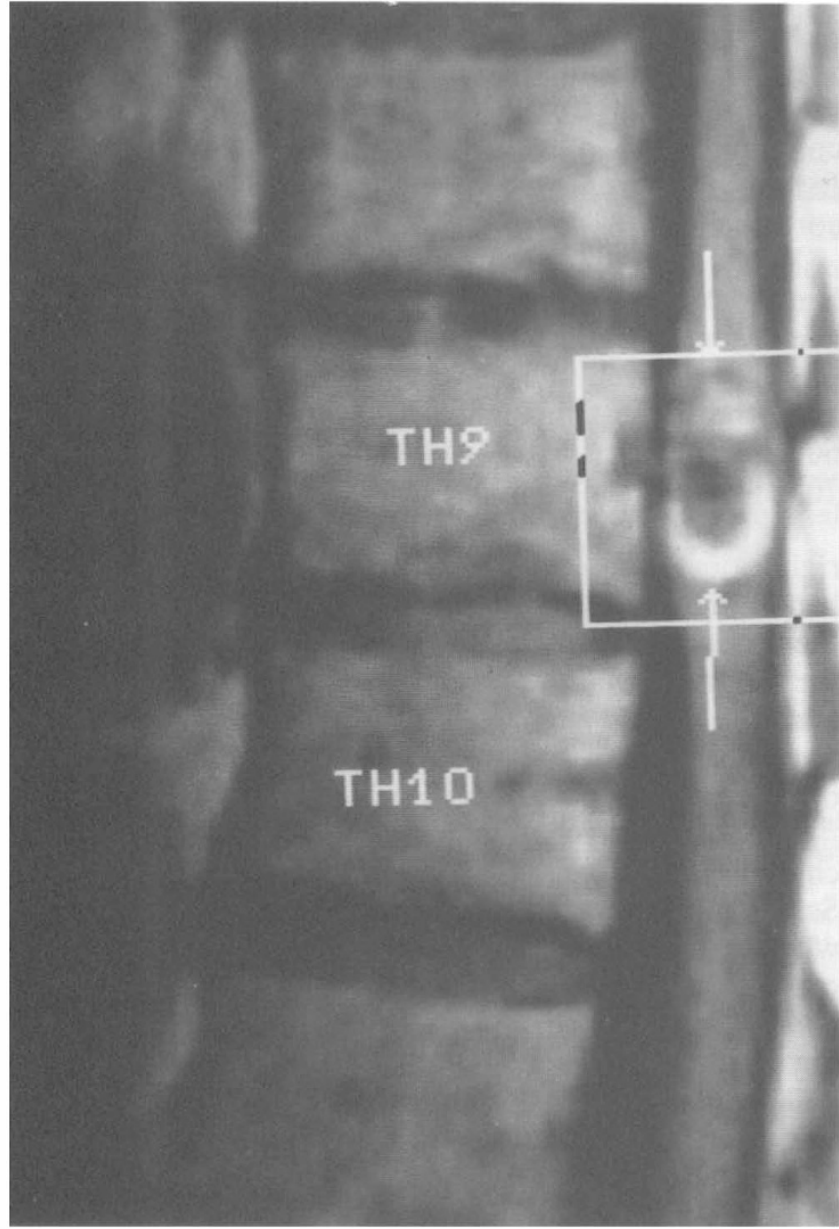

Figure 1 Case 1. MR $\mathrm{T}_{1}$-weighted image, sagittal view, showing an intramedullary mass at $\mathrm{T}_{9}$ with central low signal surrounded by areas of low and high signal intensity and aneurysmal dilatation of the aorta

and CT scan were not diagnostically helpful. MRI showed the presence of an intramedullary lesion at $\mathrm{C}_{3}-\mathrm{C}_{4}$ level with the characters of a cryptic vascular malformation and a concomitant intramedullary hematoma from $\mathrm{C}_{1}$ to $\mathrm{T}_{6}$ with cystic areas filled with blood (Figure 2).

Operation With the patient in the sitting position, she underwent a laminectomy from $\mathrm{C}_{2}$ to $\mathrm{C}_{5}$. There was mild enlargement of the spinal cord. Myelotomy disclosed a cavity containing old blood and a small vascularized nodule in the right posterolateral portion of the cavity. After irrigation of the cavity with normal saline the lesion was completely removed. Histological examination revealed the pathology to be a cavernous angioma (Figure 3 ).

The postoperative course was excellent. At follow up, the patient remains without neurological deficit, except for minor numbness of the left arm. Five months after the operation a new MRI demonstrated the remaining cavity of the hematoma (Figure 4).

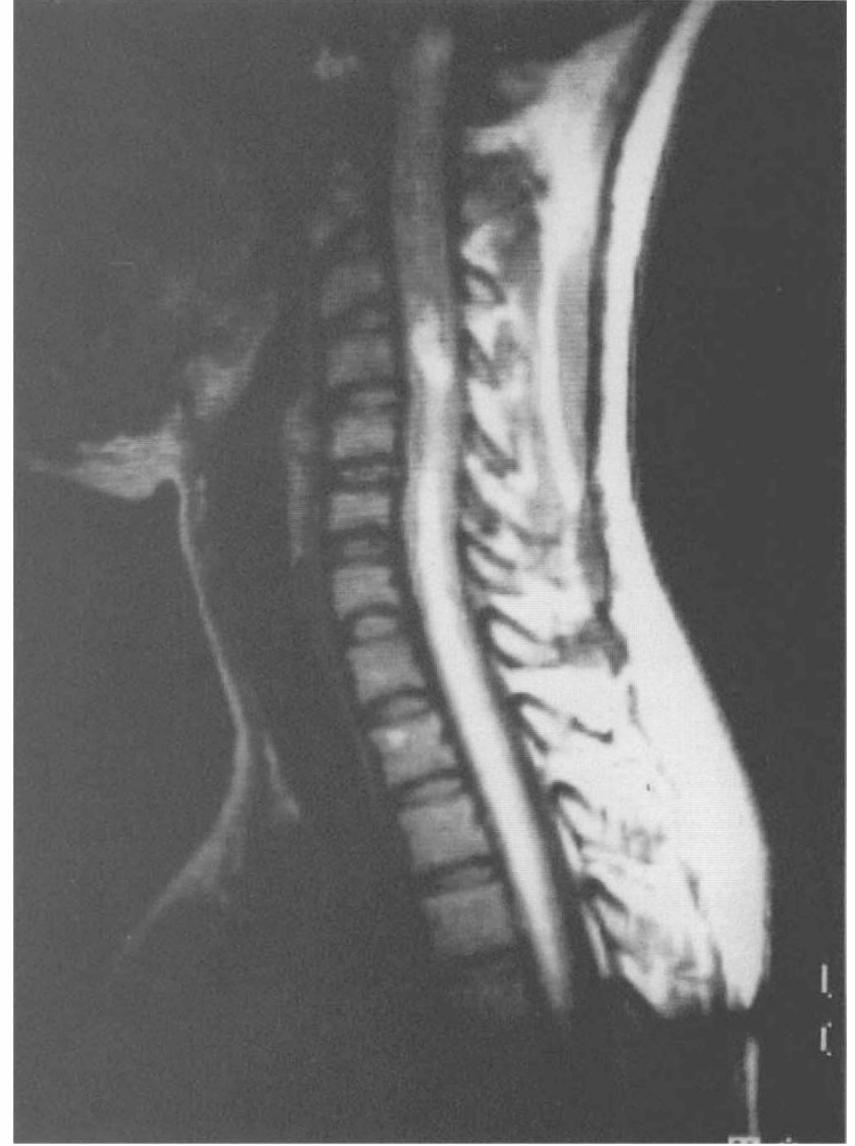

Figure 2 Case 2. MR $\mathrm{T}_{1}$-weighted image showing an intramedullary lesion at $\mathrm{C}_{3}-\mathrm{C}_{4}$ with the characters of a cryptic AVM and the concomitant hematomyelia

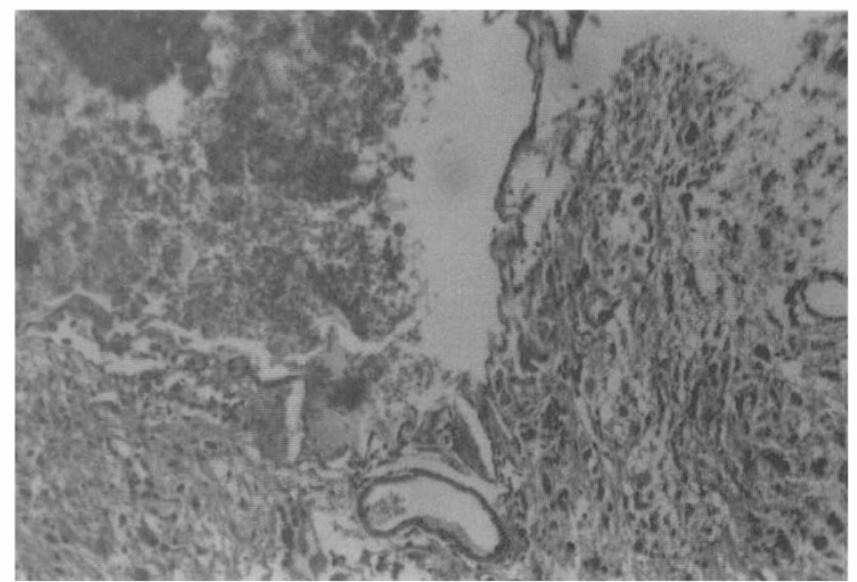

Figure 3 Case 2. Histologic appearance in light microscope of the cavernous angioma, showing the abnormal vessels surrounding by neural cells (hematoxylin-eosin $\times 40$ )

\section{Discussion}

Hematomyelia is characterized by the presence of a blood clot in the central part of the spinal cord which 


An $\begin{array}{r}\text { Non-traumatic hematomyelia } \\ \text { A Karavelis et a }\end{array}$

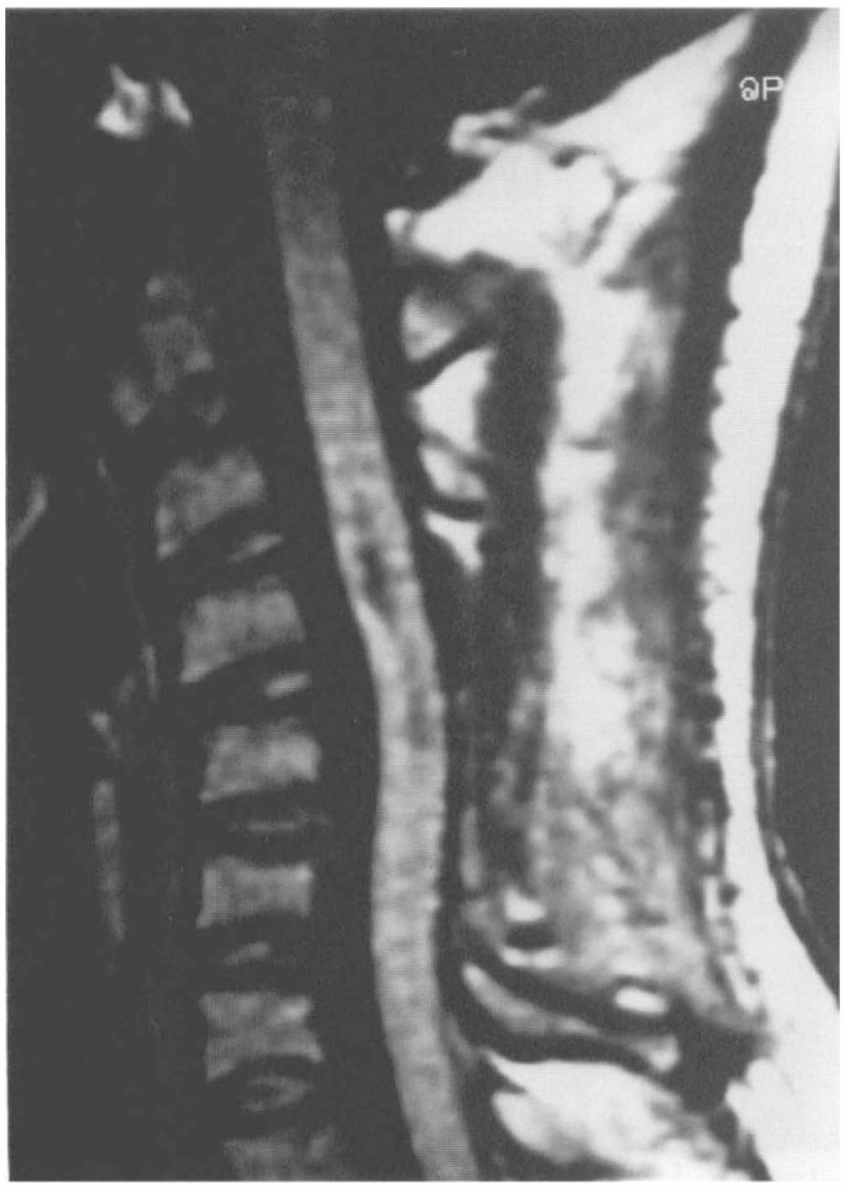

Figure 4 Case 2. Postoperative MRI showing the remaining cavity of the intramedullary hematoma

usually tends to spread longitudinally over several segments. In general, these hemorrhages occur in the gray and the neighbouring white matter. The hemorrhage involves mainly the gray matter and spreads up and down the cord rather than transversely, because of the anatomical peculiarities of the spinal cord. ${ }^{6,7}$ The size and extent of the hemorrhage varies from patient to patient. They do not commonly penetrate into the subarachnoid space.

Small cryptic vascular malformations (AVM) seem to be the main source of the bleeding. The size of these malformations in the spinal cord is usually a few millimetres in diameter, in contrast to cerebral cryptic AVM's which are larger. ${ }^{2,8}$ Hematomyelia occurring in the absence of a vascular malformation have been associated with diseases such as blood dyscrasias and bleeding disorders (hemophilia, von Willebrand's disease, factor XI deficiency, anticoagulant therapy, ${ }^{9,10}$ cardiovascular disorders (hypertension, heart failure, atherosclerosis) and various other disorders such as trauma, meningomyelitis, myelitis and arachnoiditis, tumours, compressive lesions, syringomyelia and syphilis. ${ }^{11,12}$
Most hematomyelias are localised in the cervical and thoracic spinal cord. ${ }^{13}$ Lobato et al ${ }^{14}$ reported that, 'in the brain, AVM's and cavernous angiomas constitute $75 \%$ of all cryptic vascular malformations'. In the literature, there is no similar study about spinal cord AVM's.

We believe that atherosclerosis was the cause of the hematomyelia in the first, elderly patient in whom aneurysmal dilatation of the aorta coexisted. Furthermore, it is well-known that vascular malformations have the highest incidence between the age of 30 and 60 and they are extraordinarily rare over the age of 70.3

In the second patient a cavernous angioma in the right posterolateral portion of the cavity of the hematoma was removed. Cavernous angiomas are the commonest form of vascular malformations of the spinal cord. They occur predominantly in women and the clinical course of cervical lesions is that they have both a shorter course and an increased frequency of bleeding, as occurred in our second case. $3,16,17$

In hematomyelia the clinical picture is usually characterized by the development of a BrownSequard type of syndrome, which is manifested, whether acutely by intense pain and severe neurological deficit, as in our second patient, or by progressive neurological deterioration, simulating a space occupying lesion of the spinal cord, as in our first case., 18

The commoner neuroradiological examinations (CT-scan, Myelogram and CT-myelogram) are not diagnostic. Spinal angiography is not necessary, if MRI is diagnostic and does not show the presence of abnormal vessels. MRI is the method of choice because it depicts very well the hemorrhage and the possible cause of bleeding., ${ }^{2,5}$

MRI, in our first case, revealed an intramedullary lesion that had signal characteristics of hemorrhage of variable age in both the $T_{1}$ and the $T_{2}$-weighted images, without enhancement after an intravenous infusion of contrast medium. In the second patient, MRI showed an extensive hematomyelia from $C_{1}$ to $\mathrm{T}_{6}$ and a lesion with the characteristics of a cryptic vascular malformation at $\mathrm{C}_{3}-\mathrm{C}_{4}$. The cryptic vascular malformation appears in the MRI as an intramedullary well-defined space occupying lesion, with central areas of low and high signal intensity, which may represent hemorrhage of different ages. Serpentine vessels are not demonstrated in cryptic angiomas as they are in spinal AVM's. ${ }^{19,20}$

The preoperative MRI demonstrated the lesion in our patients precisely. Especially in the second patient there were small cystic areas adjacent to the focal lesion that were filled with blood as previously mentioned by Barnwell et al. ${ }^{2}$

The surgical treatment of these lesions effectively halted the progression of the disease and prevented further deterioration. The aspiration of the hematoma and the total removal of the malformation with the aid of the operating microscope appeared to protect the patient from additonal aggravation. ${ }^{11}$ 
In conclusion, non-traumatic hematomyelia is an extremely rare condition, usually present with a Brown-Sequard type of syndrome. It is diagnosed by MRI with great accuracy. Immediate surgical treatment for the removal of the hematoma and the associated vascular malformation, if present, is considered to be the best way to halt the progression of the disease.

\section{Acknowledgements}

We thank Mrs Elli Chatsopoulou for her secretarial assistance and for her help to prepare the manuscript.

\section{References}

1 Crawford JV, Russell DS. Cryptic arteriovenous and venous hematomas of the brain. J Neurol Neurosurg Psych 1965; 19: 1 11.

2 Barnwell S et al. Cryptic vascular malformations of the spinal cord: diagnosis by magnetic resonance imaging and outcome of surgery. J Neurosurg 1990; 72: 403-407.

3 Canavero S, Pagui CA, Duca S, Bradac CB. Spinal intramedullary angiomas: literature meta-analysis. Surg Neurol 1994; 41: $381-388$.

4 Firsching R, Zanella F, Lanfermann H. The dynamica of magnetic resonance findings in patients with a spinal haematoma. Paraplegia 1993; 31: 180-185.

5 Turjman F et al. MRI of intramedullary cavernous haemangiomas. Neuroradiology 1995; 37: 297-302.

6 Leech RW, Pitha JV, Spetzler RF. Spontaneous haematomyelia: a necropsy study. J Neurol Neurosurg Psych 1991; 54: 172-174.

7 Milhoral TH et al. Histopathology hematomyelia. J Neurosurg 1991; 75: $911-915$.
8 Krayenbuhl $\mathrm{H}$, Yasargil MG, McClintock HG. Treatment of spinal cord vascular malformations by surgical excision. $J$ Neurosurg 1969; 30: $427-435$.

9 Pisani R et al. Hematomyelia during anticoagulant therapy. Surg Neurol 1985; 24: 578-580.

10 Zeidman SM, Olivi A. Cervical intramedullary hemorrhage as a result of anticoagulant therapy. J Spinal Disord 1993; 6: 456457.

11 Jellinger $\mathrm{K}$. Traumatic vascular disease of the spinal cord. In: Vinken PJ, Bruyn GW (eds). Handbook of Clinical Neurology. North-Holland Publishing company. 1972, Volume 12, pp. 556630 .

$12 \mathrm{Yu}$ JS et al. Intramedullary hemorrhage in spinal hemangioblastoma. Report of two cases. J Neurosurg 1994; 81: 937-940.

13 Koos W, Bock F. Spontaneous multiple intramedullary hematomas. J Neurosurg 1970; 32: $581-584$.

14 Lobato RD et al. Clinical, radiological and pathological spectrum of angiographically occult intracranial vascular malformations. J Neurosurg 1988; 68: 518-531.

15 Cosgrove GR et al. Cavernous angiomas of the spinal cord. $J$ Neurosurg 1988; 68: $31-36$.

16 Lee KS, Spetzler RF. Spinal cord cavernous malformation in a patient with familiar intracranial cavernous malformation. Neurosurgery 1990; 26: $877-880$.

17 Ogilvy CS, Louis DN, Ojeman RG. Intramedullary cavernous hemangiomas of the spinal cord: clinical presentation, pathological features and surgical management. Neurosurgery 1992; 31: $219-230$

18 Zentner J, Hassler W, Gawehn J, Schroth G. Intramedullary cavernous angiomas. Surg Neurol 1989; 31: 64-68.

19 Beraus R. Vascular malformations of the spinal cord. In: Vinken PJ, Bruyn GW (eds). Vascular Diseases of the Nervous System, Part II, and Handbook of Clinical Neurology, Volume 12. NorthHolland: Amsterdam, 1972, pp. 584-555.

20 Veerapen R, Sbeith AI, O'Laoire S. Surgical treatment of cryptic AVM's and associated hematoma in the brain stem and the spinal cord. J Neurosurg 1986; 65: 188 - 193. 